\title{
Distribution of genes related to Type 6 secretion system and lipooligosaccharide that induced ganglioside mimicry among Campylobacter jejuni isolated from human diarrhea in Thailand
}

Oralak Serichantalergs ${ }^{1}$, Patcharawalai Wassanarungroj ${ }^{1 *}$, Nuanpan Khemnu1', Frédéric Poly², Patricia Guerry², Ladaporn Bodhidatta' ${ }^{1}$, John Crawford $^{3}$ and Brett Swierczewski ${ }^{4}$

\begin{abstract}
Background: Campylobacter jejuni (C. jejuni) is one of the most common bacteria responsible for human gastroenteritis worldwide. The mode of human transmission is foodborne infections due to consumption of contaminated food, especially poultry. Type 6 secretion systems (T6SS) were described recently as Campylobacter virulence mechanisms. Furthermore, infection sequelae associated with neurological disorders like Guillain-Barré (GBS) and Miller Fisher (MF) syndromes can become serious health problems in some patients after Campylobacter gastroenteritis. Our objective was to determine the distribution of these virulence genes among C. jejuni isolated from stool of human diarrhea.

Methods: A total of 524 C. jejuni strains from travelers and pediatric cases of acute diarrhea in Thailand were selected for this study. All isolates belonged to one of 20 known capsule types and all were assayed by PCR for T6SS, a hemolysin co-regulated protein ( $h c p)$ gene, and GBS-associated genes (cgtA, cgtB, cstI/ ${ }_{H S 19}$ and $\left.c s t I_{H S 2}\right)$ which are involved in sialic acid production in the lipooligosaccharide (LOS) cores of C. jejuni. The distribution of these genes are summarized and discussed.

Results: Of all isolates with these 20 capsule types identified, 328 (62.6\%) were positive for $h c p$, ranging from 29.2 to $100 \%$ among 10 capsule types. The GBS-associated LOS genes were detected among 14 capsule type isolates with $24.4 \%$ and $23.3 \%$ of $C$. jejuni isolates possessed either cst $\|_{H S 19}$ or all three genes (cgtA, cgtB and $\left.c s t \|_{H S 19}\right)$, which were classified as LOS classes A and B whereas 9.2\% of C. jejuni isolates possessing cst II $_{H S 2}$ were classified as LOS class C. The $C$. jejuni isolates of LOS A, B, and C together accounted for $56.9 \%$ of the isolates among 14 different capsule types while $31.1 \%$ of all C. jejuni isolates did not possess any GBS-associated genes. No significant difference was detected from C. jejuni isolates possessing GBS-associated LOS genes among travelers and children, but changes between those with $h c p$ were significant $(p<0.05)$.
\end{abstract}

*Correspondence: PatcharawalaiW@afrims.org

1 Department of Bacterial and Parasitic Diseases, Armed Forces Research

Institute of Medical Sciences (AFRIMS), Bangkok, Thailand

Full list of author information is available at the end of the article

(c) The Author(s) 2020. This article is licensed under a Creative Commons Attribution 4.0 International License, which permits use, sharing, adaptation, distribution and reproduction in any medium or format, as long as you give appropriate credit to the original author(s) and the source, provide a link to the Creative Commons licence, and indicate if changes were made. The images or other third party material in this article are included in the article's Creative Commons licence, unless indicated otherwise in a credit line to the material. If material is not included in the article's Creative Commons licence and your intended use is not permitted by statutory regulation or exceeds the permitted use, you will need to obtain permission directly from the copyright holder. To view a copy of this licence, visit http://creativeco mmons.org/licenses/by/4.0/. The Creative Commons Public Domain Dedication waiver (http://creativecommons.org/publicdomain/ zero/1.0/) applies to the data made available in this article, unless otherwise stated in a credit line to the data. 
Conclusions: Our results suggested a high diversity of $h c p$ and GBS-associated LOS genes among capsule types of $C$. jejuni isolated from Thailand.

Keywords: Campylobacter jejuni, Capsule types, GBS, LOS, hcp, T6SS, Human diarrhea

\section{Introduction}

Campylobacter jejuni is recognized as a major pathogen of gastroenteritis worldwide [1]. As illustrated by the latest epidemiological report, Campylobacter is the main cause of foodborne illness in Europe and the United States [1]. Campylobacteriosis cases range from 14 to 57 per 100,000 per year (US and Europe, respectively). In endemic regions, particularly in Southeast Asia, campylobacteriosis cases are estimated to be 10 times higher than in Europe and the USA. This high incidence of Campylobacter is well documented in children, travelers, and foreign military personnel from Thailand [2-4]. C. jejuni is considered a zoonotic disease. The major source of contamination is through consumption of improperly prepared or stored foods containing poultry, but other sources like unpasteurized milk or water have been documented. The infectious dose can be as low as 500 to 1000 bacteria [5]. Campylobacteriosis symptoms range from mild abdominal pain and mild to no diarrhea, to severe abdominal cramping, sometimes accompanied with fever, headache, myalgia, and large volumes of mucous and bloody diarrhea that can last for several days [6].

Despite a worldwide prevalence, there is a paucity of data regarding $C$. jejuni virulence factors. Nevertheless, flagella, cytolethal distending toxin $(c d t)$, fibronectin binding protein (cadF), lipoprotein (ceuE) [7-9], and proteins involved in adherence and invasion acquired by plasmids ( $p$ Vir) [10] contribute to virulence. The Campylobacter polysaccharide capsule (CPS) is the best characterized virulence factor. Mutants deficient in CPS production demonstrated lower adherence and binding in vitro, decreased serum resistance, and reduced colonization of ferret and chicken animal models $[7,11]$. Phenotypic assays can be routinely applied for identification and classification of putative $C$. jejuni samples in laboratory settings [12]. Penner's serotyping, developed in 1980s, is a well-known serotyping scheme for C. jejuni and C. coli that is based on capsular polysaccharide [13]. Molecular assays have replaced this method for routine C. jejuni and other Campylobacter spp. identification. Analyses of different CPS sequences were utilized to develop multiplex PCR assays to distinguish more capsule types among $C$. jejuni isolates [14-17]. This capsule typing scheme correlates well to Penner serotypes because the capsule structure is the major serodeterminant [14].
In addition to the virulence factors aforementioned, additional $C$. jejuni isolates were characterized and found to possess the recently described T6SS $[18,19]$. The T6SS apparatus is composed of proteins which structurally and functionally are related to contractile components of bacteriophages [20]. The hemolysin co-regulated protein (Hcp) in Pseudomonas aeruginosa T6SS resembles the major component of the T4 phage tail base plate [21]. The T6SS contributes to bacterial pathogenesis in Pseudomonas aeruginosa [22], Vibrio cholerae [23], Salmonella enterica [24], Helicobacter hepaticus [25], Edwardsiella spp. [26], Burkoderia mallei [27], as well as in C. jejuni [19]. Campylobacter T6SS plays an important roles in host cell adhesion, invasion, and persistent colonization in vivo [19]. T6SS was reported in C. jejuni isolated from clinical, poultry, and water sources, and its distribution varied in human and animal sources from different countries [28-30]. However, the distribution and role of T6SS pathogenesis in C. jejuni from human gastroenteritis has yet to be further determined.

A myriad of post infectious sequelae were linked to $C$. jejuni infections. These include autoimmune mediated Guillain-Barré (GBS) and Miller Fisher (MF) syndromes [31]. These autoimmune diseases are provoked by mimicry of C. jejuni lipooligosaccharides (LOS) structures that contain $N$-acetyl neuraminic (sialic acid), organized into structures that resemble human gangliosides [31]. Most $C$. jejuni strains contain genes for the endogenous synthesis of $\mathrm{N}$-acetyl neuraminic acid, and there are specific genes involved in the biosynthesis of these ganglioside mimics [32]. The cgtA ( $\beta-1,4-N$-acetylgalactosyl transferase) and $\operatorname{cgt} B(\beta-1,3$-galactosyltransferase) and cstII ( $\alpha-2,3$ sialyltransferase) are required for molecular mimicry and are associated with human GBS. These genes were used as markers to screen for GBS-related strains of C. jejuni [33-35]. Multiple variants of cstII transferase were characterized in the $C$. jejuni LOS locus, including cstII $_{H S 19}$ and cstII $_{H S 2}$ [33]. The gene cstII has mono and bi-functional activities $(\alpha-2,3$ and/or $\alpha-2,3 / \alpha-$ 2,8 -sialyltranferase), and both represent different specificity of enzymes involved in the transfer of the sialic acid residue to the LOS structure [32, 36]. Genes in LOS biosynthesis loci were studied from different origins of C. jejuni, and the LOS classes A, B, and C were classified for involvement in human ganglioside mimicry. Recently, LOS classes $M$ and $R$ were reported to possess genes 
similar to LOS classes A and D, including a gene encoding a sialyltransferase (cstII) [37].

The aim of this study was to determine the distribution of genes associated with T6SS and LOS implicated in GBS among different capsule types in clinical C. jejuni isolated from stool samples of travelers and children suffering diarrhea in Thailand.

\section{Materials and methods}

\section{Clinical C. jejuni isolates and bacterial isolates}

A total of $524 C$. jejuni isolates were previously identified from stool samples collected from travelers $(\mathrm{n}=313)$ and children $(\mathrm{n}=211)$ suffering acute diarrhea in 1998-2003 and 2008-2010, respectively. C. jejuni isolated from traveler's diarrhea studies were from traveling patients seeking care at Bumrungrad International Hospital, Bangkok in 2001-2002, and from military personnel participating in Cobra Gold Exercises during 1998-2003 in Thailand. C. jejuni isolated from stool cultures of children less than 5 years old with acute diarrhea from multiple regions in Thailand during 2008-2010, were included [17]. The studies used archived frozen $C$. jejuni isolates with appropriate consent for sample donation and future research use. All links or keys to subject name, number, and personal identifiable information were destroyed. All selected $C$. jejuni isolates were stored frozen at $-70{ }^{\circ} \mathrm{C}$ at AFRIMS.

All reference C. jejuni isolates used for PCR were provided by from the National Microbiology Laboratory, Public Health Agency of Canada, Winnipeg, Manitoba, Canada. These include ATCC-43429, -43432, -43434, $-43438,-43439,-43456$, and LIO 87 for LOS class differentiation. The C. jejuni strains ATCC -43431, BH-0142, NTCT-11168, and 81-176 were reference strains for T6SS previously identified from collection strains at AFRIMS.

\section{Growth and genomic DNA purification of $C$. jejuni isolates} Campylobacter jejuni were subcultured on sheep blood agar plates, using aliquots of the frozen isolates. The plates were incubated under microaerobic conditions $\left(85 \% \mathrm{~N}_{2}, 10 \% \mathrm{CO}_{2}\right.$, and $\left.5 \% \mathrm{O}_{2}\right)$ at $42{ }^{\circ} \mathrm{C}$ overnight. Genomic DNA extraction from $C$. jejuni isolates were performed using DNeasy tissue extraction kits (Qiagen, MD, USA). The quantity and quality of DNA was estimated using a NanoDrop (Thermo scientific, MA, USA) and agarose gel electrophoresis. Gels images were visualized using ethidium bromide and a gel documentation system (Syngene, Cambridge, UK).

\section{PCR for $C$. jejuni capsule types}

Primer sets specific for $C$. jejuni capsule types were used according to previously published articles [16, 17]. In brief, four primer sets (35 capsule primer pairs) recognized a total of 47 Penner serotypes and complex types. All 524 C. jejuni genomic DNA were analyzed with four multiplex PCR sets (Alpha, Beta, Gamma, and Delta). Multiplex PCR and step cycles followed previously reported standardized procedures $[16,17]$. Primer pairs for the $331 \mathrm{bp} \operatorname{lp} x A$ gene (involved in lipid A biosynthesis) were used as an internal control for $C$. jejuni. All PCR amplification products were evaluated using a 2.0\% agarose gel (Thermo Fisher Scientific -Invitrogen, MA, USA) in $0.5 \times$ Tris-Borate EDTA buffer at 150 volts for $60 \mathrm{~min}$. The DNA bands were visualized and photographed under UV light after the gel was stained with ethidium bromide. The amplification products were interpreted accordingly as described in [16].

\section{PCR detection for T6SS gene}

An hcp sequence from C. jejuni T6SS was obtained from GenBank, accession number JX436640. Primers specific to the $h c p$ gene were designed in this study using Primer3 software [38]. The $h c p$ forward (hcp_1) and reverse (hcp_2) primer sequences are 5'-CAAATGCGC AAGAGTCAAGT- $3^{\prime}$ and 5'-TAAGCTTTGCCCTCT CTCCA-3', respectively. Primers specific for $l p x A$ was included in these PCR as an internal control. The DNA extracts of C. jejuni ATCC 43431 and clinical isolates BH-01-142 were used as positive controls for $h c p$ [19]. The negative control strains were C. jejuni NCTC 11168 and 81-176 [18, 28]. Multiplex PCR was performed on a Nexus GX2 thermal cycler (Eppendorf, NY, USA) using AmpliTaq Gold DNA Polymerase (Thermo Fisher Scientific, MA, USA). The PCR step cycles for $h c p$ were 1 cycle for $94{ }^{\circ} \mathrm{C}$ for $5 \mathrm{~min}, 28$ cycles for $94{ }^{\circ} \mathrm{C}$ for $1 \mathrm{~min}$, $52{ }^{\circ} \mathrm{C} 1 \mathrm{~min}, 72{ }^{\circ} \mathrm{C}$ for $1 \mathrm{~min}$, and a final cycle at $72{ }^{\circ} \mathrm{C}$ for 10 min. A 100 bp DNA ladder (Thermo Fisher Scientific, MA, USA) was applied as a molecular marker. All PCR amplification products were evaluated on a $2.0 \%$ agarose gel (Invitrogen) at 150 volts for $60 \mathrm{~min}$. The DNA bands were visualized and photographed under UV light after the gel was stained with ethidium bromide. The estimated size of the hcp amplified products was $133 \mathrm{bp}$.

\section{PCR detection for GBS-associated LOS genes}

Four primer pairs specific for the LOS genes implicated in ganglioside mimicry: $\operatorname{cgtA}(\beta-1,4-$ $N$-acetylgalactosyltransferase $), \quad \operatorname{cgtB} \quad(\beta-1,3-$ galactosyltransferase $), \quad$ cstII $(\alpha-2,3$ sialyltransferase, and/or cstII ( $\alpha-2,3 / \alpha-2,8$ sialyltransferase), were used, as previously described [35]. Multiplex PCR was performed on a thermal cycler using AmpliTaq Gold DNA Polymerase. The PCR step cycles were: $94{ }^{\circ} \mathrm{C}$ for $5 \mathrm{~min}$, $94{ }^{\circ} \mathrm{C}$ for $1 \mathrm{~min}, 53{ }^{\circ} \mathrm{C}$ (cgtA and cgtB, cstII ${ }_{H S 19}$ and cstI$I_{H S 2}$ ) for $1 \mathrm{~min}$, and $72{ }^{\circ} \mathrm{C}$ for $1 \mathrm{~min}$ for $28 \mathrm{cycles}$, with a final $10 \mathrm{~min}$ extension at $72{ }^{\circ} \mathrm{C}$. Positive controls for 
the GBS-associated LOS genes in PCR were genomic DNA from C. jejuni ATCC 43432 (LOS-A1), ATCC 43438 (LOS-A2), ATCC 43456 (LOS-B1), ATCC 43439 (LOS-B2), NCTC 11168, ATCC 43429 (LOS-C), LIO 87 (LOS-D), and ATCC 43434 (LOS-E) to determine LOS classes A-E in C. jejuni as previously described [32, 39]. The internal PCR control was C. jejuni specific lpxA. All PCR amplification products were applied and evaluated on a $3.0 \%$ agarose-1000 gel (Invitrogen) at 150 volts for $100 \mathrm{~min}$. A $100 \mathrm{bp}$ DNA ladder was used as a molecular marker. The DNA bands were visualized and photographed under UV light after the gel was stained with ethidium bromide. The sizes of amplified products from

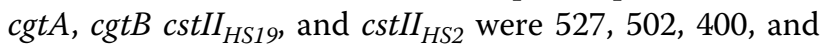
$417 \mathrm{bp}$, respectively. Primers of the four genes, and one internal control gene herein distinguished control isolates as follows: ATCC 43432 and ATCC 43456, representing LOS classes $\mathrm{A} 1$ and $\mathrm{B} 1$, were positive for all three genes $c g t A, c g t B$, and $c s t I I_{H S 19}$ whereas the control strains ATCC 43438 and ATCC 43449 representing LOS classes A2 and B2 were only positive for gene cstII $_{H S 19}$. Two control strains, NCTC 11168 and ATCC 43429 both representing LOS class $\mathrm{C}$, were positive for only cstII $_{H S 2}$. Two control strains LIO 87 and ATCC 43434 representing LOS classes D and E, respectively were negative for all four genes studied. Thus, $C$. jejun $i$ isolates possessing $\operatorname{cstII}_{H S 19}$ or all three genes (cgtA, cgtB, and $\left.\operatorname{cst} I_{H S 19}\right)$ were characterized as either LOS class A or class B, whereas isolates positive for only $c s t I I_{H S 2}$ were classified as LOS class $\mathrm{C}$. Isolates negative for all genes were classified as non-LOS classes A, B, or C.

\section{Sequencing of PCR amplified products and analysis}

Amplification products of selected clinical $C$. jejuni isolates positive for $h c p, c g t A, c g t B, c s t I I_{H S 19}$, and $c s t I I_{H S 2}$ were purified for sequencing using the Wizard SV gel and PCR clean-up system (Promega, WI, USA). After purification, the amplified products for $h c p$ and the four GBSassociated LOS genes were submitted for sequencing (Macrogen, South Korea). The amplified products of hcp, $\operatorname{cgtA}, \operatorname{cgtB}, \operatorname{cstII}_{H S 19}$, and $c s t I I_{H S 2}$ were edited and assembled by Sequencher version 5.0 (Gene Codes Corporation, MI, USA). The nucleotide sequences were analyzed with BLAST (http://www.ncbi.nlm.nih.gov/BLAST).

\section{The association of $C$. jejuni capsule types, $h c p$, and GBS-associated LOS genes}

The distribution of $h c p$ and GBS-associated LOS genes detected in C. jejuni isolated from travelers and child diarrhea cases were tested for statistical differences using Fishers exact test from GraphPad software [40]. A $p$ value $<0.05$ was considered statistically significant.

\section{Results}

Clinical C. jejuni isolates and capsule type distribution

A total of 524 archived $C$. jejuni isolates were previously characterized for capsule typing [16], and these comprised 20 capsule types. More than $82 \%(n=429)$ of all isolates belonged to eight major capsule types: HS4, HS2, HS23/36, HS53, HS5/31, HS8/17, HS3, and HS1/44, as indicated in Fig. 1. The remaining 95 isolates, ranging from 1 to $3.8 \%$ were capsule types HS37, HS15, HS42, HS10, HS9, HS6/7, and HS12; and $<1 \%$ for capsule types HS52, HS18, HS21, HS11, and HS19, each. Additionally, 92 C. jejuni-HS4 isolates gave the following HS4 subtypes: $\mathrm{HS}_{c p x A B}(\mathrm{n}=64,12.2 \%) ; \mathrm{HS}_{c p x A}(\mathrm{n}=21,4.0 \%)$, and $\mathrm{HS}_{c p x B}(\mathrm{n}=7,1.3 \%)$.

Different proportions and significance of capsule types among $C$. jejuni isolates from travelers and children were observed in these two populations (Table 1). The prevalence of capsule types in these isolates more frequent in travelers compared to children: $\mathrm{HS}_{c p x A B}$, HS23/36, HS53, and HS15. In contrast, C. jejuni HS2, HS8/17, HS1/44, and HS9 showed greater prevalence in children compared to travelers.

\section{Distribution of the $h c p$ gene}

Figure 2 represents the amplified 133-bp PCR products of the $h c p$ gene detected in 13 selected C. jejuni isolated from different capsule types in travelers and children. As shown in Table 2, the $h c p$ gene was detected in $62.6 \%(328 / 524)$ of all C. jejuni isolates but less frequently in travelers than children $55.6 \%(174 / 313)$ versus $73.0 \%(154 / 211)$, respectively $(p=0.0001)$. Only $C$.

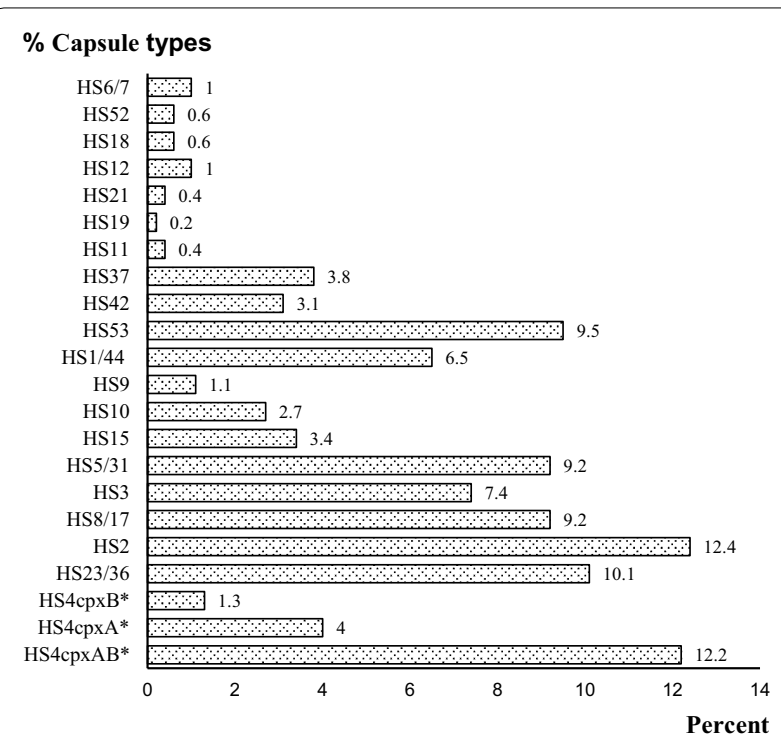

Fig. 1 Distribution of capsule types in 524 C. jejuni isolated from Thailand. ${ }^{*} \mathrm{HS} 4 \mathrm{cpxA}, \mathrm{HS} 4 \mathrm{cpxB}$, and HS4cpxAB are HS4cpx subtypes 
Table 1 Distribution of 524 C. jejuni isolates and capsule types from two studies groups

\begin{tabular}{|c|c|c|c|c|c|}
\hline Capsule types & $\begin{array}{l}\text { Travelers } \\
(\mathrm{n}=313)\end{array}$ & $\%$ & $\begin{array}{l}\text { Children } \\
(n=211)\end{array}$ & $\%$ & $p$-value ${ }^{b}$ \\
\hline $\mathrm{HS} 4_{c p x}^{\mathrm{a}}$ & 63 & 20.1 & 29 & 13.7 & NS \\
\hline $\mathrm{HS} 4_{C p \times A B}$ & 47 & 15.0 & 17 & 8.1 & 0.0203 \\
\hline $\mathrm{HS} 4_{c p \times A}$ & 9 & 2.9 & 12 & 5.7 & NS \\
\hline $\mathrm{HS} 4_{c p \times B}$ & 7 & 2.2 & 0 & 0 & N/A \\
\hline HS2 & 23 & 7.3 & 42 & 19.9 & 0.0001 \\
\hline HS23/36 & 48 & 15.3 & 5 & 2.4 & 0.0001 \\
\hline HS53 & 43 & 13.7 & 7 & 3.3 & 0.0001 \\
\hline $\mathrm{HS} 5 / 31$ & 23 & 7.3 & 25 & 11.8 & NS \\
\hline HS8/17 & 18 & 5.8 & 30 & 14.2 & 0.0017 \\
\hline HS3 & 18 & 5.8 & 21 & 10 & NS \\
\hline HS1/44 & 14 & 4.5 & 20 & 9.5 & 0.0292 \\
\hline HS37 & 13 & 4.2 & 7 & 3.3 & NS \\
\hline HS15 & 16 & 5.1 & 2 & 0.9 & 0.0127 \\
\hline HS42 & 16 & 5.1 & 0 & 0 & N/A \\
\hline HS10 & 7 & 2.2 & 7 & 3.3 & NS \\
\hline HS9 & 1 & 0.3 & 5 & 2.4 & 0.0413 \\
\hline $\mathrm{HS6/7}$ & 0 & 0 & 5 & 2.4 & N/A \\
\hline HS12 & 2 & 0.6 & 3 & 1.4 & NS \\
\hline HS52 & 3 & 1 & 0 & 0 & N/A \\
\hline HS18 & 1 & 0.3 & 2 & 0.9 & NS \\
\hline HS21 & 1 & 0.3 & 1 & 0.5 & NS \\
\hline HS11 & 2 & 0.6 & 0 & 0 & N/A \\
\hline HS19 & 1 & 0.3 & 0 & 0 & N/A \\
\hline Total & 313 & 100.0 & 211 & 100.0 & \\
\hline
\end{tabular}

NS not significance, N/A not applicable

${ }^{a} \mathrm{HS4}_{c p x}$ composed of $\mathrm{HS}_{c p \times A B^{\prime}} \mathrm{HS} 4_{c p \times A}$ and $\mathrm{HS} 4_{c p \times A B}$

${ }^{b}$ Fisher exacts test

jejuni HS8/17-hcp positive isolates were detected more often in children $(86.7 \% ; 26 / 30)$ than in travelers $(22.2 \%$; $4 / 18$ ), while we did not observe any $h c p$ significance in other capsule type isolates from travelers and children. The prevalence of $h c p$ detected varied from 28.6 to $100 \%$ in isolates of 10 capsule types: HS4cpx (all subtypes), HS23/36, HS2, HS8/17, HS3, HS5/31, HS1/44, HS15, HS10, and HS9, and no hcp genes were detected from the remaining $10 \mathrm{C}$. jejuni capsule types: HS53, HS42, HS37, HS11, HS12, HS18, HS19, HS21, HS52, and HS6/7 (Fig. 3).

\section{Distribution of GBS associated-LOS genes}

Percent distribution of four GBS-associated LOS genes are described in Table 2. There were no significant differences between a given gene, or group of genes, among travelers compared to children, except for $C$. jejuni isolates that harbored $\operatorname{cgtB}$ and $\operatorname{cstII} I_{H S 19}(p<0.0001)$. C. jejuni isolates that harbored $c s t I I_{H S 19}(24.4 \%)$ alone, or three genes for $\operatorname{cgt} A, \operatorname{cgtB}$ and $\operatorname{cstII} I_{H S 19}(23.3 \%)$, were the two most common patterns in this study. Six variants of $29 C$. jejuni isolates possessed the following gene variations: (cgtA, cgtB), (cgtA, cgtB, cstII $H_{H S 19}$, and $\left.\operatorname{cst} I I_{H S 2}\right)$, and $\left(\operatorname{cstII}_{H S 19}, \operatorname{cstII}_{H S 2}\right)$. The $\operatorname{cgt} B$ and $\left(\operatorname{cgt} A, \operatorname{cgt} B, \operatorname{cstII} I_{H S 19}\right.$ $\left.c s t I I_{H S 2}\right)$ groups were observed at a very low frequency from 0.2 to $2.1 \%$, respectively. In addition, $C$. jejuni isolates harboring $c s t I I_{H S 2}$ and $c g t B+c s t I I_{H S 19}$ were detected at $9.2 \%$ and $6.5 \%$, respectively. Overall, 163 of $524(31.1 \%)$ of C. jejuni isolates lacked all four GBS-associated LOS genes, and 78 and 85 were isolates obtained from travelers and children, respectively (Fig. 3). All 163 C. jejuni isolates belonged to 18 different capsule types: $\mathrm{HS} 1 / 44$, HS11, HS12, HS18, HS15, HS21, HS23/36, HS31, HS37, HS4cp $x A$, HS4cp $x B$, HS 42, HS52, HS53, HS6/7, HS8/17, and HS9, whereas all five capsule types isolates: HS9, HS 12, HS6/7, HS18 and HS52 always lacked all four GBSassociated LOS genes in this study. Figure 4 depicts amplified PCR products corresponding to $\operatorname{cgt} A, \operatorname{cgt} B$, $c s t I I_{H S 19}$, and $c s t I I_{H S 2}$ from selected C. jejuni isolated from travelers and children in this study.

\section{Association of capsule types of $C$. jejuni isolates with $h c p$ and GBS-associated LOS genes}

Figure 3 depicts a comparison of $h c p$, GBS-associated LOS genes and each of $C$. jejuni capsule type in this study: $h c p$, (cgtA, cgtB and cstII $\left.{ }_{H S 19}\right), c s t I I_{H S 19}$, and cstI$I_{H S 2}$. A bar graph displays the percent of $h c p$, and two line graphs represent percent of GBS-associated LOS genes: $\left[\left(\operatorname{cgtA}, \operatorname{cgtB}\right.\right.$ and $\left.\left.c s t I I_{H S 19}\right), c s t I I_{H S 19}\right]$ and $c s t I I_{H S 2}$.

A high percentage (60-100\%) of $C$. jejuni isolates in capsule types $\mathrm{HS}_{c p x A B}, \mathrm{HS} 4_{c p x A}, \mathrm{HS} 4_{c p x B}, \mathrm{HS} 10, \mathrm{HS} 2$, and HS1/44 were associated with $h c p$ and GBS-associated classes A and B LOS genes. In contrast, C. jejuni HS3, HS23/36 and HS15 isolates possessed a lower percentage of LOS class A and B as compared to $h c p$. C. jejuni HS8/17 and HS9 isolates harbored only $h c p$ but did not have LOS classes A and B. C. jejuni HS42, HS53, HS37, HS21, and HS19 isolates had GBS-associated LOS genes represented LOS class A and B, but not $h c p$ (dot line versus bar graph displays).

In the line graph display, three $C$. jejuni isolates capsule types: HS2, HS8/17, and HS15 possessed only cstII $\mathrm{HS2}_{2}$, representing LOS class $\mathrm{C}$ as $32.3 \%, 37.5 \%$, and $27.8 \%$, respectively. These $C$. jejuni isolates from the three capsule types also harbored $h c p$, whereas $C$. jejuni isolates from HS2, HS3, HS1/44, HS53, and HS15 possessed both LOS classes A, B, or C.

\section{Discussion}

In this study, HS4 and HS2 were the most common $C$. jejuni capsule types from traveler and pediatric diarrhea cases, respectively. These two capsule types were the two most common Penner serotypes in global human $C$. 


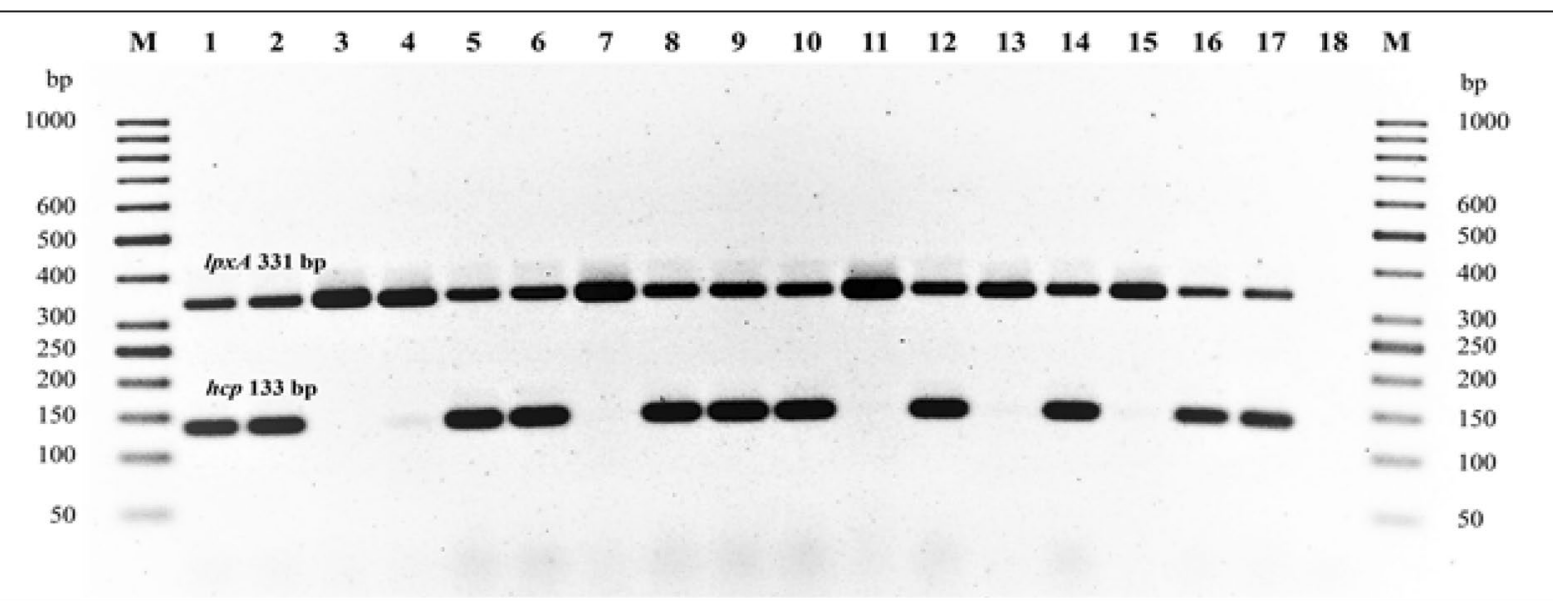

Fig. 2 Gel electrophoresis of amplified products of $h c p$ (133 bp) and IpxA (331 bp). Lane M: 100 bp DNA maker; Lane 1, 2: positive controls ATCC43431, BH-01-142; Lane 3, 4: negative controls NCTC 11168 and 81-176; Lane 5-11: clinical C. jejuni isolated from travelers; BH-01-270 (HS3, hcp positive), BH-01-39 (HS2, hcp positive), BH-01-6 (HS19, hcp negative), CG-02-3018, (HS4 cpxAB, hcp positive), CG-3-3030 (HS 23/36, hcp positive), CG-03-4020 (HS23/36, hcp positive) and CG-98-U-22 (HS3, hcp negative); Lane 12-17: clinical C. jejuni isolated from children; CR-08-181 (HS2, hcp positive), KR-08-132 (HS53, hcp negative), PN-08-207 (HS2, hcp positive), PN-08-083 (HS4, hcp positive), PN-08-128 (HS23/36, hcp positive), and PN-08-230 (HS3, hcp positive); Lane 18: negative control (nuclease-free water)

Table 2 Distribution of C. jejuni, hcp, and GBS-associated LOS genes in travelers and children

\begin{tabular}{|c|c|c|c|c|c|c|c|c|}
\hline C. jejuni traits & LOS & Total (n) & $\%$ & Travelers (n) & $\%$ & Children (n) & $\%$ & $p$-value \\
\hline C.jejuni, hcp & - & $328(524)$ & 62.6 & $174(313)$ & 55.6 & $154(211)$ & 73.0 & 0.0001 \\
\hline $\mathrm{HS} 8 / 17, h c p$ & - & $30(48)$ & 62.5 & $4(18)$ & 22.2 & $26(30)$ & 86.7 & 0.0001 \\
\hline C. jejuni, cgtA, cgtB, cstII HS19 & $A, B$ & $122(524)$ & 23.3 & 79 (313) & 25.2 & $43(211)$ & 20.4 & NS \\
\hline C.jejuni, cstII ${ }_{H S 19}$ & $A, B$ & $128(524)$ & 24.4 & $71(313)$ & 22.7 & $51(211)$ & 24.2 & NS \\
\hline C. jejuni, cstII ${ }_{H S 2}$ & C & $48(524)$ & 9.2 & $19(313)$ & 13.1 & $29(211)$ & 13.7 & NS \\
\hline C. jejuni, cgtB & N/A & $6(524)$ & 1.3 & $6(313)$ & 1.9 & $0(211)$ & 0 & $\mathrm{~N} / \mathrm{A}$ \\
\hline C. jejuni, cgtA, cgtB & $N / A$ & $1(524)$ & 0.2 & $0(313)$ & 0 & $1(211)$ & 0.5 & $\mathrm{~N} / \mathrm{A}$ \\
\hline C. jejuni, cgtB, cstII ${ }_{H S 19}$ & N/A & $34(524)$ & 6.5 & $32(313)$ & 10.2 & $2(211)$ & 1.0 & 0.0001 \\
\hline C. jejuni, $c s t I_{H S 19}, C s t I_{H S 2}$ & $\mathrm{~N} / \mathrm{A}$ & $6(524)$ & 1.1 & $6(313)$ & 1.9 & $0(211)$ & 0 & $\mathrm{~N} / \mathrm{A}$ \\
\hline C. jejuni, cgtB, cstII ${ }_{H S 19}, c{ }^{\prime} I_{H S 2}$ & N/A & $11(524)$ & 2.1 & $11(313)$ & 3.5 & $0(211)$ & 0 & $\mathrm{~N} / \mathrm{A}$ \\
\hline C. jejuni, cgtA, cgtB, cstII ${ }_{H S 19}, c s t \|_{H S 2}$ & N/A & $5(524)$ & 0.9 & $5(313)$ & 1.6 & $0(211)$ & 0 & $\mathrm{~N} / \mathrm{A}$ \\
\hline
\end{tabular}

NS not significance, N/A not applicable

a Fisher exact test was calculated for significance, comparing travelers and children

jejuni isolates, confirming previous reports [41]. Other capsule types exhibited varied distribution among isolates from travelers and children. The study of traveler's diarrhea isolates included C. jejuni, of which $>80 \%$ were obtained from stool samples of military personnel attending annual exercises from different locations in Thailand. A point-source of infection of an identical C. jejuni clone isolated from the same type of food consumed might attribute a distribution of one capsule type over the others (e.g., HS53, HS23/36). The different capsule types seen in travelers may be affected by temporal variations during the exercises of military personnel, and by the contaminated food consumed by travelers at the visiting sites (e.g., resort or hotel), but this was not seen in isolates from children in which samples were collected over a 2 year period. Although $C$. jejuni isolates from these two studies were from more than 5 years apart, the five most common capsule types from traveler isolates (HS4, HS2, HS5/31, HS8/17, and HS3) and Thai children isolates (HS2, HS 8/17, HS4, HS5/31, and HS3) were comparable to capsule serotypes of global C. jejuni isolates reported previously [41].

T6SS are bacteriophage-like structures which secrete effector molecules into eukaryotic cells or other bacteria 


\section{Percent}

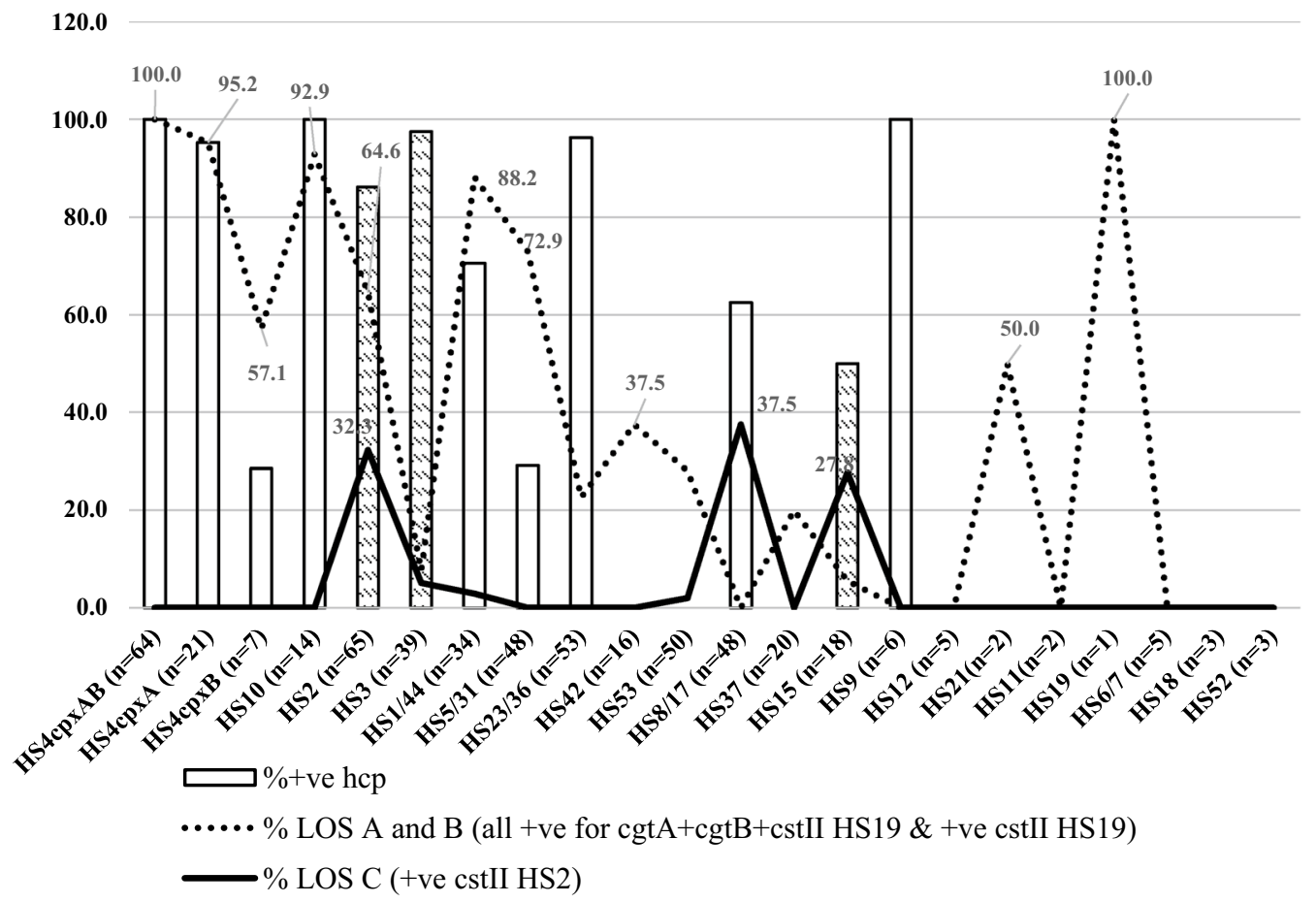

Fig. 3 Distribution of $h c p$ and GBS-associated LOS genes amongst C. jejuni capsule types. A bar graph and two line graphs represent the percent distribution of hcp and LOS (class A or B) and class C genes, respectively among numbers of C. jejuni isolates of each capsule type. A bar graph with a filled pattern show C. jejuni capsule types associated both hcp, LOS classes A, B, and C

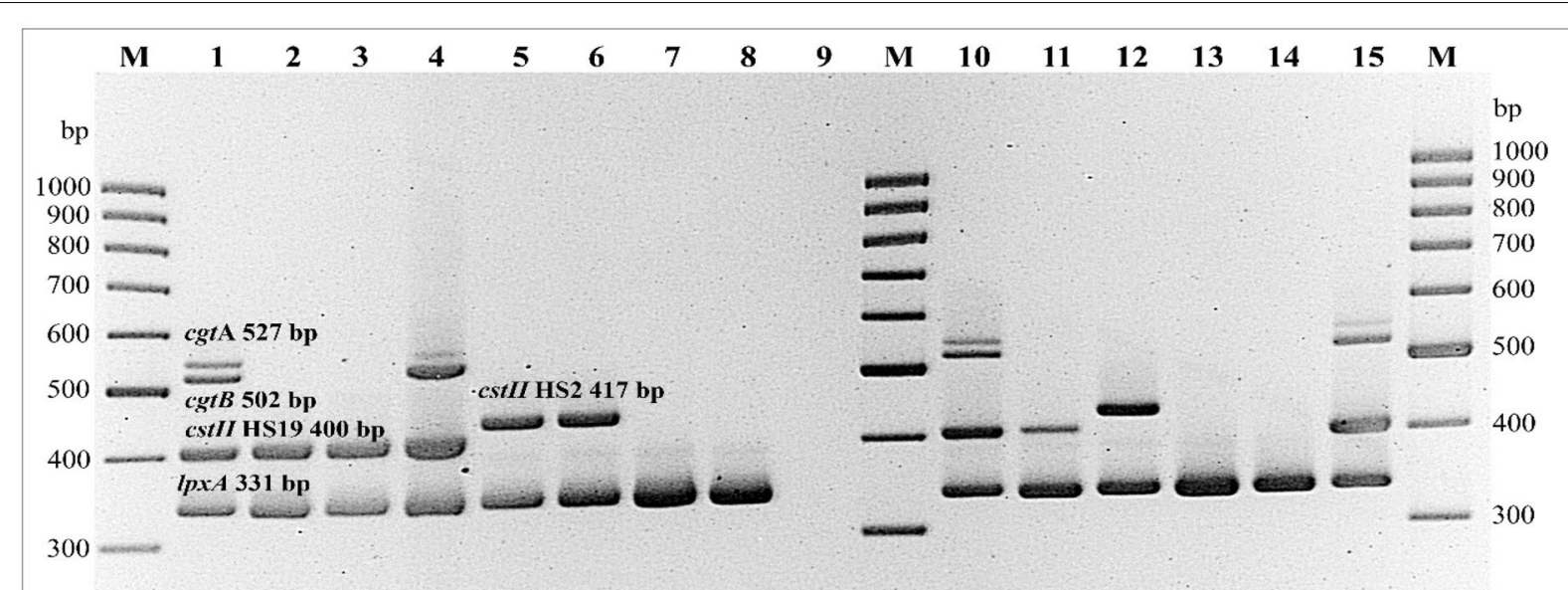

Fig. 4 Gel electrophoresis of amplified products of GBS-associated LOS genes from selected C. jejuni isolates from travelers and children. PCR

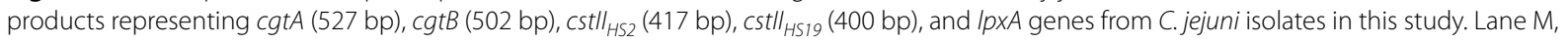
100 bp DNA maker; Lane 1, ATCC 43432 (LOS class A1); Lane 2, ATCC 43438 (LOS class A2); Lane 3, ATCC 43449 (LOS class B2); Lane 4, ATCC 43456 (LOS class B1); Lane 5, 6: NCTC 11168 and ATCC 43429 (LOS class C); Lane 7, LIO87 (LOS class D); Lane 8, ATCC 43434 (LOS class E); Lane 9, negative control (nuclease-free water); Lane 10, 11, and 13, amplified product of selected C. jejuni isolates from travelers CG-01-09510 (HS2), CG-03-4049 (HS23/36), and CG-99-8135 (HS23/36), respectively; Lane 12, 14, and 15, amplified products of selected C. jejuni isolates from children, SR-08-150 (HS2), BH-01-193 (HS15), and KR-08-207 (HS4 ${ }_{c p \times A B}$ ), respectively 
$[20,42]$. The presence of a secreted effector, the $h c p$ needle protein, in culture supernatants suggested contribution of this protein to virulence of $C$. jejuni strains [18, 19]. The $h c p$ gene was used as a surrogate for the presence of T6SS in $C$. jejuni. Using this screening method on different $C$. jejuni isolates, heterogeneous results were reported. Harrison et al. [28] demonstrated the prevalence of $h c p$ in $60.6 \%$ and $33.3 \%$ from clinical C. jejuni isolates in Vietnam and Thailand, respectively. However, only three human $C$. jejuni isolates from Thailand were studied. None of the T6SS genes were detected in $34 C$. jejuni isolated from human diarrheal cases in Pakistan [29]. In our study, $h c p$ was found in $62.6 \%$ of the 524 cases, and this proportion was similar to studied isolates in Vietnam (60.6\%) and Egypt (57.6\%) [28, 43].

The $h c p$ gene was associated with 10 capsule types of C. jejuni isolates whereas a variable $h c p$ prevalence was reported by others. Sainato et al. [43] reported a $h c p$ gene prevalence of $33.3 \%$ in HS2 isolates, but up to $86.2 \%$ was noted in this study. Moreover, numbers of $h c p-C$. jejuni isolates were more significant in children than travelers (22.2\%, 86.7\%; $p=0.0001)$, especially in HS8/17 isolates. Different distributions of the $h c p$ gene were also observed among HS4cpx isolates (28.5\% of $\mathrm{HS}_{c p x B}, 92.5 \% \mathrm{HS}_{c p x A}$, and $100 \%$ of $\left.\mathrm{HS}_{(p p x A B}\right)$. Our study was limited in that we tested for only one gene marker in T6SS, while the T6SS locus composed of at least 13 ORFs [18]. A fully-functional T6SS was only reported in two C. jejuni strains in published articles $[18,19]$. Further investigation on more conserved genes in the T6SS locus and expression of their encoded proteins may implicate the contribution of T6SS to Campylobacter virulence.

$H c p$ was previously reported to be associated with bloody diarrhea [28]. We found no significant association between $h c p$ and $C$. jejuni isolates of any specific capsule type. This is similar to the finding by Agnetti et al. [44], where no significant difference in the clinical manifestations and the course of disease between patient with hcppositive and -negative $C$. jejuni isolates. Similarly, the presence of $h c p$ gene in C. jejuni and C. coli from stool did not have any significant association to clinical outcome in Egyptian children [43].

Campylobacter jejuni and their LOS classes could be distinguished by different LOS biosynthesis locus, as referenced by others [32, 33, 39]. Parker et al, reported orf locus $7 \mathrm{ab}$ and $7 \mathrm{c}$ to encode for $c s t I I$ and cstIII, respectively. In our study, the primers for four genes, cgtA, cgtB, cstII $I_{H S 19}$ and $c s t I I_{H S 2}$, were used following Nachamkin, et al. [35]. The amplified products representing clinical C. jejuni of different capsule types, shown in Fig. 4, were sequenced and analyzed with BLASTn to identify each gene. The alignment matched within locus of $\operatorname{cgtA}$, cgtB, cstII, and cstIII, respectively. In this study, we focused on LOS classes A to $C$ that can cause sialylated LOS synthesis resulting in ganglioside mimicry, which are crucial for ganglioside mimicry from various $C$. jejuni strains, as previous reported [32, 39, 45-47]. The GBS genes for LOS classes A, B are predominately amongst C. jejuni HS19 isolates [47]. All $524 C$. jejuni isolates were associated with gastroenteritis (GI). CstII $I_{H S 19}$ was previously reported in GBS-HS19 isolates more than in GBS-non HS19 isolates [35]. In this study, almost 50\% (23.3 and $24.4 \%$ in Table 2) of the GI-isolates were classified as LOS classes A or B, but only one isolate was HS19 serotype. GBS was commonly associated with HS1/44-, HS2-, and HS4-C. jejuni antecedent infections in the Netherlands, whereas HS19-, HS 23/36-, and HS41-C. jejuni were associated with GBS in Bangladesh, Japan, and South Africa [47]. Our study demonstrated a high prevalence of HS1/44, HS2, HS23/36, and $\mathrm{HS}_{c p x}$, but the HS41 serotype was not detected from the GI-isolates. Distribution of LOS classes A, B, and C in C. jejuni has been previously demonstrated, as $17 \%, 32 \%$, and $19 \%$, respectively, but there are data linked to only 5 Penner serotypes from these C. jejuni isolates [47]. Parker et al. [39] reported that $64 \%$ of non-GBS associated isolates were in LOS classes (A, B, or $\mathrm{C})$, and this is comparable to our study that $56.9 \%$ of $C$. jejuni isolated were in LOS classes A, B, and C. A limitation of our study was that the primers used for LOS associated GBS genes could not amplify gene targets to differentiate LOS subclasses $\mathrm{A} 1, \mathrm{~A} 2, \mathrm{~B} 1$ and B2, and other LOS loci diversity [37, 39, 48, 49]. More primers specific to other LOS classes should be included to characterize these $C$. jejuni isolates in more depth, especially in $63 \mathrm{C}$. jejuni isolates with unclassified LOS locus.

Differences in association of CPS and MLST types were also seen among these $C$. jejuni strains compared to published studies. Heikema et al. [47] observed that HS23/36 isolates from GBS patients were associated with a MLST clonal complex (CC)-403, -42 , and -206 , but most of HS23/36-GI isolates in this study were MLST CC-52 (>90\%). Similarly most of GBS-HS2 isolates in the Heikema study were MLST CC-21, but our GI-HS2 isolates were MLST-CC-464 (83\%) and unassigned CC (27\%). HS4-GBS and enteritis isolates were MLST CC-48, -61, -206, and -508 (Heikema), while most of GI-isolates in our study were MLST-52, -460 , and unassigned CC (data not shown). This suggested there was a selective and divergent clone lineage of $C$. jejuni GBS- and GI-strains; however, many other factors likely also contribute to Campylobacter virulence. 


\section{Conclusion}

This data provides information on the diversity of $h c p$ and LOS genes implicated with GBS-associated C. jejuni capsule types in travelers and children, as compared to other studies. A high prevalence of T6SS and GBS-associated LOS genes was found in certain $C$. jejuni capsule types: HS4 $4_{c p x A B}, \mathrm{HS} 4_{c p x A}$, HS2, HS1/44, and HS10, which may suggest an increase fitness of these Campylobacter since most isolates contained hcp and LOS classes A, B, or $C$ genes, which are crucial for survival in the host and promote the synthesis of sialylated LOS. However, variations in clonal strains, different biological LOS functions, as well as host and environment factors, may be involved in triggering an autoimmune response and need further study. The understanding of global capsule types, antigenic epitopes, and virulence determinants may contribute towards future development of targeted therapeutics against $C$. jejuni diarrhea.

\section{Abbreviations}

T6SS: Type 6 secretion system; GBS: Guillain-Barré syndrome; MF: Miller Fisher; LOS: Lipooligosaccharide; MLST: Multilocus sequence typing; CC: Clonal complex; GI: Gastroenteritis; CPS: Capsule polysaccharide; ORF: Open reading frame.

\section{Acknowledgements}

Authors would like to thank staff members of the Department of Enteric Diseases, AFRIMS on their expertise in sample collection, microbiological processing and laboratory supports. Most of all, we are grateful to Jason Moses from National Microbiology Laboratory, Public Health Agency of Canada, Winnipeg, Manitoba, Canada for providing reference C. jejuni strains used in this study. We also thanks to the department personnel on administrative supports of the project.

\section{Authors' contributions}

OS, PG, and FP designed the concept of this study. OS, PW, and NK designed and performed experiments. OS and PF analyzed data and drafted manuscripts. LB, BES, and JC provided research material and critical revision of this article. All authors read and approved the final manuscript.

\section{Funding}

This study was supported by Military Infectious Disease Researches Program. Walter Reed Army Institute of Research, USA and by the U.S. Naval Medical Research and Development Command Work Unit 6000.RAD1.DA3.A0308.

\section{Availability of data and materials}

All the supporting the findings are presented in the manuscript.

\section{Ethic approval and consent to participate}

Studies in this report were approved by Bamrungrad IRB, The Ethical Review Committee for Research in Human Subjects, Thai Ministry of Public Health, and WRAIR IRB. The consents to participate were signed by subjected for future use studies.

\section{Consent for publication}

Not applicable.

\section{Competing interests}

Material has been reviewed by the Walter Reed Army Institute of Research. There is no objection to its presentation and/or publication. The opinions or assertions contained herein are the private views of the author, and are not to be construed as official, or as reflecting true views of the Department of the Army or the Department of Defense. The investigators have adhered to the policies for protection of human subjects as prescribed in AR 70-25."Authors are US military service members (BES and JC) and employees of the US government (PG, FP, LB). This work was prepared as part of official duties. Title 17 U.S.C. 105 provides that 'copyright protection under this title is not available for any work of the United States Government.'Title 17 U.S.C. 101 defines a U.S. Government work as work prepared by a military service member or employee of the U.S. Government as part of that person's official duties".

\section{Author details}

${ }^{1}$ Department of Bacterial and Parasitic Diseases, Armed Forces Research Institute of Medical Sciences (AFRIMS), Bangkok, Thailand. ${ }^{2}$ Enteric Diseases Department, Naval Medical Research Center, Silver Spring, MD, USA. ${ }^{3}$ US Army Medical Research Institute of Chemical Defense, Aberdeen, MD, USA. ${ }^{4}$ Bacterial Diseases Branch, Walter Reed Army Institute of Research, Silver Spring, MD, USA.

Received: 31 January 2020 Accepted: 4 April 2020

Published online: 09 April 2020

\section{References}

1. Kaakoush NO, Castano-Rodriguez N, Mitchell HM, Man SM. Global epidemiology of Campylobacter infection. Clin Microbiol Rev. 2015;28(3):687-720

2. Bodhidatta L, Vithayasai N, Eimpokalarp B, Pitarangsi C, Serichantalergs O, Isenbarger DW. Bacterial enteric pathogens in children with acute dysentery in Thailand: increasing importance of quinolone-resistant Campylobacter. Southeast Asian J Trop Med Public Health. 2002;33(4):752-7.

3. Sanders JW, Isenbarger DW, Walz SE, Pang LW, Scott DA, Tamminga C, et al. An observational clinic-based study of diarrheal illness in deployed United States military personnel in Thailand: presentation and outcome of Campylobacter infection. Am J Trop Med Hyg. 2002;67(5):533-8.

4. Taylor DN, Echeverria P, Pitarangsi C, Seriwatana J, Bodhidatta L, Blaser MJ. Influence of strain characteristics and immunity on the epidemiology of Campylobacter infections in Thailand. J Clin Microbiol. 1988;26(5):863-8.

5. Black RE, Levine MM, Clements ML, Hughes TP, Blaser MJ. Experimental Campylobacter jejuni infection in humans. J Infect Dis. 1988;157(3):472-9.

6. Blaser MJ. Epidemiologic and clinical features of Campylobacter jejuni infections. J Infect Dis. 1997;176(Suppl 2):S103-5.

7. Bacon DJ, Szymanski CM, Burr DH, Silver RP, Alm RA, Guerry P. A phasevariable capsule is involved in virulence of Campylobacter jejuni 81-176. Mol Microbiol. 2001;40(3):769-77.

8. Bang DD, Nielsen EM, Scheutz F, Pedersen K, Handberg K, Madsen M. PCR detection of seven virulence and toxin genes of Campylobacter jejuni and Campylobacter coli isolates from Danish pigs and cattle and cytolethal distending toxin production of the isolates. J Appl Microbiol. 2003:94(6):1003-14

9. Monteiro MA, Baqar S, Hall ER, Chen YH, Porter CK, Bentzel DE, et al. Capsule polysaccharide conjugate vaccine against diarrheal disease caused by Campylobacter jejuni. Infect Immun. 2009;77(3):1128-36.

10. Bacon DJ, Alm RA, Burr DH, Hu L, Kopecko DJ, Ewing CP, et al. Involvement of a plasmid in virulence of Campylobacter jejuni 81-176. Infect Immun. 2000;68(8):4384-90.

11. Nemelka KW, Brown AW, Wallace SM, Jones E, Asher LV, Pattarini D, et al. Immune response to and histopathology of Campylobacter jejuni infection in ferrets (Mustela putorius furo). Comp Med. 2009;59(4):363-71.

12. Lastovica AJ, le Roux E. Efficient isolation of campylobacteria from stools. J Clin Microbiol. 2000;38(7):2798-9.

13. Penner $J$ L, Hennessy JN. Passive hemagglutination technique for serotyping Campylobacter fetus subsp. jejuni on the basis of soluble heat-stable antigens. J Clin Microbiol. 1980;12(6):732-7.

14. Karlyshev AV, Linton D, Gregson NA, Lastovica AJ, Wren BW. Genetic and biochemical evidence of a Campylobacter jejuni capsular polysaccharide that accounts for Penner serotype specificity. Mol Microbiol. 2000;35(3):529-41.

15. Karlyshev AV, Wren BW, Moran AP. Campylobacter jejuni capsular polysaccharide. In: Nachamkin I, Szymanski CM, Blaser MJ, editors. Campylobacter. 3rd ed. Washington D.C: ASM Press; 2008. p. 505-22.

16. Poly F, Serichantalergs $O$, Kuroiwa J, Pootong P, Mason C, Guerry P, et al. Updated Campylobacter jejuni capsule PCR multiplex typing system and 
its application to clinical isolates from South and Southeast Asia. PLoS ONE. 2015;10(12):e0144349.

17. Poly F, Serichatalergs O, Schulman M, Ju J, Cates CN, Kanipes M, et al. Discrimination of major capsular types of Campylobacter jejuni by multiplex PCR. J Clin Microbiol. 2011:49(5):1750-7.

18. Bleumink-Pluym NM, van Alphen LB, Bouwman LI, Wosten MM, van Putten JP. Identification of a functional type VI secretion system in Campylobacter jejuni conferring capsule polysaccharide sensitive cytotoxicity. PLoS Pathog. 2013:9(5):e1003393.

19. Lertpiriyapong K, Gamazon ER, Feng Y, Park DS, Pang J, Botka G, et al. Campylobacter jejuni type VI secretion system: roles in adaptation to deoxycholic acid, host cell adherence, invasion, and in vivo colonization. PLoS ONE. 2012;7(8):e42842.

20. Basler M, Pilhofer M, Henderson GP, Jensen GJ, Mekalanos JJ. Type VI secretion requires a dynamic contractile phage tail-like structure. Nature. 2012;483(7388):182-6.

21. Lossi NS, Dajani R, Freemont P, Filloux A. Structure-function analysis of HsiF, a gp25-like component of the type VI secretion system, Pseudomonas aeruginosa. Microbiology. 2011;157(Pt 12):3292-305.

22. Mougous JD, Cuff ME, Raunser S, Shen A, Zhou M, Gifford CA, et al. A virulence locus of Pseudomonas aeruginosa encodes a protein secretion apparatus. Science. 2006;312(5779):1526-30.

23. Das S, Chaudhuri K. Identification of a unique IAHP (IcmF associated homologous proteins) cluster in Vibrio cholerae and other proteobacteria through in silico analysis. In silico Biol. 2003;3(3):287-300.

24. Blondel CJ, Jimenez JC, Contreras I, Santiviago CA. Comparative genomic analysis uncovers 3 novel loci encoding type six secretion systems differentially distributed in Salmonella serotypes. BMC Genomics. 2009;10:354.

25. Bartonickova L, Sterzenbach T, Nell S, Kops F, Schulze J, Venzke A, et al. Hcp and VgrG1 are secreted components of the Helicobacter hepaticus type VI secretion system and VgrG1 increases the bacterial colitogenic potential. Cell Microbiol. 2013;15(6):992-1011.

26. Zheng J, Leung KY. Dissection of a type VI secretion system in Edwardsiella tarda. Mol Microbiol. 2007;66(5):1192-206.

27. Schell MA, Ulrich RL, Ribot WJ, Brueggemann EE, Hines HB, Chen D, et al. Type VI secretion is a major virulence determinant in Burkholderia mallei. Mol Microbiol. 2007;64(6):1466-85.

28. Harrison JW, Dung TT, Siddiqui F, Korbrisate S, Bukhari H, Tra MP, et al. Identification of possible virulence marker from Campylobacter jejuni isolates. Emerg Infect Dis. 2014:20(6):1026-9.

29. Siddiqui F, Champion O, Akram M, Studholme D, Eqani SA, Wren BW, et al. Molecular detection identified a type six secretion system in Campylobacter jejuni from various sources but not from human cases. J Appl Microbiol. 2015;118(5):1191-8.

30. Ugarte-Ruiz M, Stabler RA, Dominguez 542 L, Porrero MC, Wren BW, Dorrell $\mathrm{N}$, et al. Prevalence of type VI secretion system in Spanish Campylobacter jejuni isolates. Zoonoses public health. 2015;62(7):497-500.

31. Endtz HP, Ang CW, van Den Braak N, Duim B, Rigter A, Price $\amalg$, et al. Molecular characterization of Campylobacter jejuni from patients with Guillain-Barre and Miller Fisher syndromes. J Clin Microbiol. 2000;38(6):2297-301.

32. Gilbert M, Karwaski MF, Bernatchez S, Young NM, Taboada E, Michniewicz $J$, et al. The genetic bases for the variation in the lipo-oligosaccharide of the mucosal pathogen, Campylobacter jejuni. Biosynthesis of sialylated ganglioside mimics in the core oligosaccharide. J Biol Chem. 2002;277(1):327-37

33. Gilbert M, Brisson JR, Karwaski MF, Michniewicz J, Cunningham AM, Wu Y, et al. Biosynthesis of ganglioside mimics in Campylobacter jejuni $\mathrm{OH} 4384$. Identification of the glycosyltransferase genes, enzymatic synthesis of model compounds, and characterization of nanomole amounts by 600 mhz (1)h and (13)c NMR analysis. J Biol Chem. 2000;275(6):3896-906.

34. Linton D, Gilbert M, Hitchen PG, Dell A, Morris HR, Wakarchuk WW, et al. Phase variation of a beta-1,3 galactosyltransferase involved in generation of the ganglioside GM1-like lipo-oligosaccharide of Campylobacter jejuni. Mol Microbiol. 2000;37(3):501-14.

35. Nachamkin I, Liu J, Li M, Ung H, Moran AP, Prendergast MM, et al. Campylobacter jejuni from patients with Guillain-Barre syndrome preferentially expresses a GD(1a)-like epitope. Infect Immun. 2002;70(9):5299-303.

36. Godschalk PC, Kuijf ML, Li J, St Michael F, Ang CW, Jacobs BC, et al. Structural characterization of Campylobacter jejuni lipooligosaccharide outer cores associated with Guillain-Barre and Miller Fisher syndromes. Infect Immun. 2007;75(3):1245-54.

37. Parker CT, Gilbert M, Yuki N, Endtz HP, Mandrell RE. Characterization of lipooligosaccharide-biosynthetic loci of Campylobacter jejuni reveals new lipooligosaccharide classes: evidence of mosaic organizations. J Bacteriol. 2008;190(16):5681-9.

38. Untergasser A, Cutcutache I, Koressaar T, Ye J, Faircloth BC, Remm M, et al. Primer3-new capabilities and interfaces. Nucleic Acids Res. 2012:40(15):e115.

39. Parker CT, Horn ST, Gilbert M, Miller WG, Woodward DL, Mandrell RE. Comparison of Campylobacter jejuni lipooligosaccharide biosynthesis loci from a variety of sources. J Clin Microbiol. 2005;43(6):2771-81.

40. GraphPad software. http://www.graphpad.com/quickcalcs/Contingenc y1.cfm. Accessed 25 Apr 2017.

41. Pike BL, Guerry P, Poly F. Global distribution of penner serotypes: a systematic review. PLoS ONE. 2013;8(6):e67375.

42. Silverman JM, Brunet YR, Cascales E, Mougous JD. Structure and regulation of the type VI secretion system. Annu Rev Microbiol. 2012;66:453-72.

43. Sainato R, ElGendy A, Poly F, Kuroiwa J, Guerry P, Riddle MS, et al. Epidemiology of Campylobacter infections among children in Egypt. Am J Trop Med Hyg. 2018;98(2):581-5.

44. Agnetti J, Seth-Smith HMB, Ursich S, et al. Clinical impact of the type VI secretion system on virulence of Campylobacter species during infection. BMC Infect Dis. 2019;19(1):237.

45. Guerry P, Ewing CP, Hickey TE, Prendergast MM, Moran AP. Sialylation of lipooligosaccharide cores affects immunogenicity and serum resistance of Campylobacter jejuni. Infect Immun. 2000;68(12):6656-62.

46. Guerry P, Szymanski CM, Prendergast MM, Hickey TE, Ewing CP, Pattarini $\mathrm{DL}$, et al. Phase variation of Campylobacter jejuni 81-176 lipooligosaccharide affects ganglioside mimicry and invasiveness in vitro. Infect Immun. 2002:70(2):787-93.

47. Heikema AP, Islam Z, Horst-Kreft D, Huizinga R, Jacobs BC, Wagenaar JA, et al. Campylobacter jejuni capsular genotypes are related to Guillain-Barre syndrome. Clin Microbiol Infect. 2015;21 (9):852.e1-9.

48. Koga M, Gilbert M, Takahashi M, Li J, Koike S, Hirata K, et al. Comprehensive analysis of bacterial risk factors for the development of Guillain-Barre syndrome after Campylobacter jejuni enteritis. J Infect Dis. 2006:193(4):547-55

49. Godschalk PC, Heikema AP, Gilbert M, Komagamine T, Ang CW, Glerum $J$, et al. The crucial role of Campylobacter jejuni genes in anti-ganglioside antibody induction in Guillain-Barre syndrome. J Clin Invest. 2004;114(11):1659-65.

\section{Publisher's Note}

Springer Nature remains neutral with regard to jurisdictional claims in published maps and institutional affiliations. 